\title{
Deterministic Time-Reversible Thermostats : Chaos, Ergodicity, and the Zeroth Law of Thermodynamics
}

\author{
William Graham Hoover, Ruby Valley Research Institute \\ Highway Contract 60 Box 601, Ruby Valley Nevada USA 89833 ; \\ Julien Clinton Sprott, Department of Physics \\ University of Wisconsin Madison, Wisconsin 53706 ; \\ Puneet Kumar Patra, Advanced Technology Development Center \\ Department of Civil Engineering, Indian Institute of Technology \\ Kharagpur, West Bengal, India 721302 .
}

(Dated: July 17, 2018)

\begin{abstract}
The relative stability and ergodicity of deterministic time-reversible thermostats, both singly and in coupled pairs, are assessed through their Lyapunov spectra. Five types of thermostat are coupled to one another through a single Hooke's-Law harmonic spring. The resulting dynamics shows that three specific thermostat types, Hoover-Holian, Ju-Bulgac, and Martyna-Klein-Tuckerman, have very similar Lyapunov spectra in their equilibrium four-dimensional phase spaces and when coupled in equilibrium or nonequilibrium pairs. All three of these oscillator-based thermostats are shown to be ergodic, with smooth analytic Gaussian distributions in their extended phase spaces ( coordinate, momentum, and two control variables ). Evidently these three ergodic and time-reversible thermostat types are particularly useful as statistical-mechanical thermometers and thermostats. Each of them generates Gibbs' universal canonical distribution internally as well as for systems to which they are coupled. Thus they obey the Zeroth Law of Thermodynamics, as a good heat bath should. They also provide dissipative heat flow with relatively small nonlinearity when two or more such bath temperatures interact and provide useful deterministic replacements for the stochastic Langevin equation.

PACS numbers: 05.20.Jj, 47.11.Mn, 83.50.Ax
\end{abstract}

Keywords: Chaotic Dynamics, Time Reversibility, Temperature, Thermostats, Chaos, Zeroth Law 


\section{DETERMINISTIC TIME-REVERSIBLE THERMOSTATS AND ERGODICITY}

In the early days of equilibrium many-body simulation, intercomparisons of results from constant-temperature Monte Carlo with those from constant-energy molecular dynamics were indirect, requiring the conversion of isothermal $N V T$ data to isoenergetic $N V E$ data. Only for hard disks and hard spheres, where temperature and energy are proportional, are the two ensembles identical ${ }^{1}$.

Shuichi Nosé provided a conceptual breakthrough linking temperature $T$ and energy $E$ by discovering an isothermal canonical-ensemble molecular dynamics ${ }^{2,3}$. He started out by including an additional time-reversible "time-scaling variable" $s$ in his novel Hamiltonian :

$$
\mathcal{H}_{\text {Nosé }} \equiv\left[K(p) / s^{2}\right]+\Phi(q)+\left(p_{s}^{2} / 2 M\right)+(\# k T / 2) \ln \left(s^{2}\right) \text {. }
$$

Here \# is the number of degrees of freedom, including $s$, and $p_{s}$ is the momentum conjugate to $s . M$ is a free parameter. Getting to the canonical ensemble from Nosé's Hamiltonian equations of motion requires just two more steps ; [ i ] "scaling the time", multiplying all of Nosé's time derivatives by $s$ :

$$
\{\dot{q} \rightarrow s \dot{q} ; \dot{p} \rightarrow s \dot{p}\} ; \dot{s} \rightarrow s \dot{s} ; \dot{p}_{s} \rightarrow s \dot{p}_{s}
$$

then [ ii ] replacing all the scaled momenta $\{(p / s)\}$ by $\{p\}$. Nosé showed that the resulting distribution for the $\{q, p\}$ is Gibbs' canonical distribution.

Starting instead with the "Nosé-Hoover" equations of motion" ${ }^{4}-\underline{\underline{7}}$,

$$
\{\dot{q}=(p / m) ; \dot{p}=F(q)-\zeta p\} ; \dot{\zeta}=\sum^{\#=N D}\left[\left(p^{2} / m k T\right)-1\right] / \tau^{2},
$$

Hoover showed that Gibbs' canonical distribution, with $s$ absent and with \# $=N D$ not including that extraneous $s$ variable, is a stationary solution of the extended phase-space continuity equation ,

$$
(\partial f / \partial t)=0=-\sum^{\#}(\partial f \dot{q} / \partial q)-\sum^{\#}(\partial f \dot{p} / \partial p)-(\partial f \dot{\zeta} / \partial \zeta)
$$

Here $\zeta$ is a "friction coefficient" proportional to Nosé's $p_{s}$ and the free parameter $\tau$ takes the place of Nosé's parameter $M$. The stationary distribution function for the Nosé-Hoover motion equations is canonical in the $\{q, p\}$ and Gaussian in the friction coefficient $\zeta$ :

$$
f(q, p, \zeta) \propto e^{-\mathcal{H} / k T} e^{-\tau^{2} \zeta^{2} / 2} ; \mathcal{H}=\Phi(q)+K(p)
$$


The relaxation time $\tau$ controls the decay rate of velocity fluctuations in a $D$-dimensional $N$-body Hamiltonian system.

In order for averages using the Nosé-Hoover equations to agree with canonical-ensemble averages, it is necessary in principle that the dynamics be "ergodic", meaning that it must reach all of the $\{q, p\}$ phase-space states. Of course in practice only a representative sampling of such states can be achieved. Whether or not the motion equations are "ergodic" in this sense, capable of reaching all of the states described by Gibbs' canonical ensemble, depends upon the details of the underlying Hamiltonian $\mathcal{H}(q, p)$. For a simple harmonic oscillator Hoover pointed out that neither the four original unscaled Nosé equations nor the three Nosé-Hoover motion equations are ergodic :

$$
\begin{gathered}
\dot{q}=+\left(p / s^{2}\right) ; \dot{p}=-q ; M \ddot{s}=\dot{p}_{s}=\left[\left(p^{2} / s^{3}\right)-(2 / s)\right] ;[\text { Nosé, Not Ergodic }] . \\
\dot{q}=+p ; \dot{p}=-q-\zeta p ; \dot{\zeta}=\left[p^{2}-1\right] / \tau^{2} ;[\text { Nosé - Hoover, Not Ergodic ] . }
\end{gathered}
$$

These Nosé equations are singular while the Nosé-Hoover equations are not.

With the relaxation time $\tau$ equal to unity, numerical Nosé-Hoover calculations show that about five percent of the initial conditions drawn from the Gaussian distribution $e^{-q^{2} / 2} e^{-p^{2} / 2} e^{-\zeta^{2} / 2}$ are chaotic, making up a "chaotic sea" in the $(q, p, \zeta)$ space which is penetrated by an infinite number of holes. The remaining $95 \%$ of initial conditions generate regular periodic toroidal solutions which fill in these holes. Evidently this Nosé-Hoover thermostated oscillator is far from "ergodic" [ where in this case an ergodic solution would have a smooth analytic Gaussian density throughout $(q, p, \zeta)$ space ] :

$$
f_{\text {Gibbs }}(q, p, \zeta) \propto e^{\left(-q^{2} / 2\right)} e^{\left(-p^{2} / 2\right)} e^{\left(-\zeta^{2} / 2\right)}[\text { Ergodic }]
$$

In 1990 Bulgac and Kusnezov reiterated the usefulness of the phase-space continuity equation in formulating more complicated motion equations ( with two or three thermostating control variables ). Shortly thereafter they were able to use this approach to fill out the complete Gibbs' distribution for prototypical Hamiltonian systems like the harmonic oscillator and the two-well "Mexican Hat" problems $\underline{\underline{8}-10}$.

There is no foolproof test for ergodicity. The only reliable diagnostic for space-filling ergodicity is the Lyapunov spectrum $11-19$. If this spectrum, which measures the long-timeaveraged sensitivity of the dynamics to initial conditions, is not only chaotic, but also the same for all initial conditions, the system is likely ergodic. For the harmonic oscillator 
this means that all $(q, p)$ oscillator states, all the way to infinity, will eventually occur. In nonergodic systems the long-time-averaged spectrum depends upon the initial conditions. In ergodic systems the long-time spectrum is always the same. By 1996 there were three known simple types of differential equations $[\mathrm{HH}=$ Hoover-Holian, JB = Ju-Bulgac, MKT = Martyna-Klein-Tuckermann ] which were thought to produce ergodicity in a onedimensional harmonic oscillator $\underline{9,17}-\underline{19}$. In addition to propagating the phase variables $(q, p)$ all three included two additional control variables $(\zeta, \xi)$ capable of generating Gibbs' canonical distribution. For the simple harmonic oscillator these three models take the form :

$$
\begin{aligned}
& \left\{\dot{q}=p ; \dot{p}=-q-\zeta p-\xi p^{3} ; \dot{\zeta}=p^{2}-T ; \dot{\xi}=p^{4}-3 T p^{2}\right\}[\mathrm{HH}] ; \\
& \left\{\dot{q}=p ; \dot{p}=-q-\zeta^{3} p-\xi p^{3} ; \dot{\zeta}=p^{2}-T ; \dot{\xi}=p^{4}-3 T p^{2}\right\}[\mathrm{JB}] ; \\
& \left\{\dot{q}=p ; \dot{p}=-q-\zeta p ; \dot{\zeta}=p^{2}-T-\xi \zeta ; \dot{\xi}=\zeta^{2}-T\right\}[\mathrm{MKT}] .
\end{aligned}
$$

So far no one-thermostat oscillator system ( with just a single control variable ) has been shown to be ergodic though it certainly is possible that such a one will be discovered. We explicitly include the temperature $T$ in all of these models in order to set the stage for nonequilibrium systems incorporating both "cold" and "hot" degrees of freedom. It is worth pointing out that not all two-thermostat oscillator systems are ergodic. Patra and Bhattacharya showed that their very reasonable set of doubly-thermostated oscillator equations is not ergodic $\frac{14,19}{2}$

$$
\left\{\dot{q}=p-\xi q ; \dot{p}=-q-\zeta p ; \dot{\zeta}=p^{2}-T ; \dot{\xi}=q^{2}-T\right\}[\mathrm{PB}]
$$

Our work here has three different aspects. First we explore the equilibrium Lyapunov instability which facilitates the ergodicity of all three thermostats. In the equilibrium case, where the phase-space distribution is a smooth Gaussian, the Lyapunov instability embedded in that Gaussian has a totally different multifractal character. We show that the equilibrium HH, JB, and MKT thermostats obey the Zeroth Law when coupled with one another or with other Hamiltonian systems. These thermostats can provide Gibbs' complete canonical distribution provided that any internal energy barriers are not too large relative to $k T$. Second we consider nonequilibrium cases, where heat flows between thermostats with the thermostat temperatures set at different values. The nonequilibrium dynamics is still ergodic but the phase-space distribution is no longer a smooth Gaussian. It is instead an intimate multifractal combination of the attractor-repellor pairs common to macroscopic time-reversible, 
but dissipative, systems. We also illustrate the application of ergodic thermostats to kinetic barrier-crossing problems. Last of all, we summarize the lessons learned in this study of three different sorts of applications of our ergodic time-reversible thermostats.

\section{LYAPUNOV EXPONENTS-THERMOSTATED HARMONIC OSCILLATORS}

The most convincing evidence for the lack of ergodicity with one thermostat variable, and its lack or presence in two-thermostat systems, is the Lyapunov spectrum. Here we concentrate on thermostated oscillators, the prototypical "difficult" case. A harmonic-oscillator system of four ordinary differential equations, such as the HH, JB, MKT, and PB sets for $(\dot{q}, \dot{p}, \dot{\zeta}, \dot{\xi})$ has four such exponents $\left(\lambda_{1}, \lambda_{2}, \lambda_{3}, \lambda_{4}\right)$. These exponents describe the deformation of an infinitesimal hypersphere or hypercube in the four-dimensional phase space which contains the motion. Shimada and Nakashima ${ }^{11}$, as well as Benettin's group $\frac{12}{2}$ described general approaches to determining the spectrum of exponents.

In an $n$-dimensional space their algorithms require the solution of $n+1$ sets of $n$ equations. In addition to a "reference trajectory" the equations describe the motion of an associated orthonormal set of $n$ comoving basis vectors, centered on the reference trajectory and locating $n$ nearby "satellite trajectories" in the $n$-dimensional space. The first Lyapunov exponent gives the time-averaged rate at which two neighboring solutions of the equations diverge from one another, $\left\langle\dot{\delta}(t) \simeq e^{\lambda_{1} t}\right\rangle$. The rate at which the area defined by three nearby solutions ( the reference and two others ) diverges ( or converges ) $\simeq e^{\lambda_{1} t} e^{\lambda_{2} t}$ defines $\lambda_{2}$, while the rate at which the volume defined by four solutions and the hypervolume defined by all five of them define $\lambda_{3}$ and $\lambda_{4}$. Although these exponents are typically "paired" for Hamiltonian systems, with

$$
\left[\lambda_{1} \equiv\left\langle\lambda_{1}(t)\right\rangle=-\left\langle\lambda_{4}(t)\right\rangle ; \lambda_{2} \equiv\left\langle\lambda_{2}(t)\right\rangle=-\left\langle\lambda_{3}(t)\right\rangle=0\right] \rightarrow \sum_{1}^{4} \lambda_{i} \equiv 0
$$

with the sum total equal to zero, as implied by Liouville's Theorem, none of the twothermostat systems is Hamiltonian so that this instantaneous symmetry is missing in the time-dependent exponents $\{\lambda(t)\}$. But because the equations of motion are time-reversible the time-averaged spectra $\{\lambda\}$ are symmetric.

For an ergodic system the long-time-averaged spectrum of exponents should be independent of the initial conditions. In practice, for the harmonic-oscillator system, a few hundred 


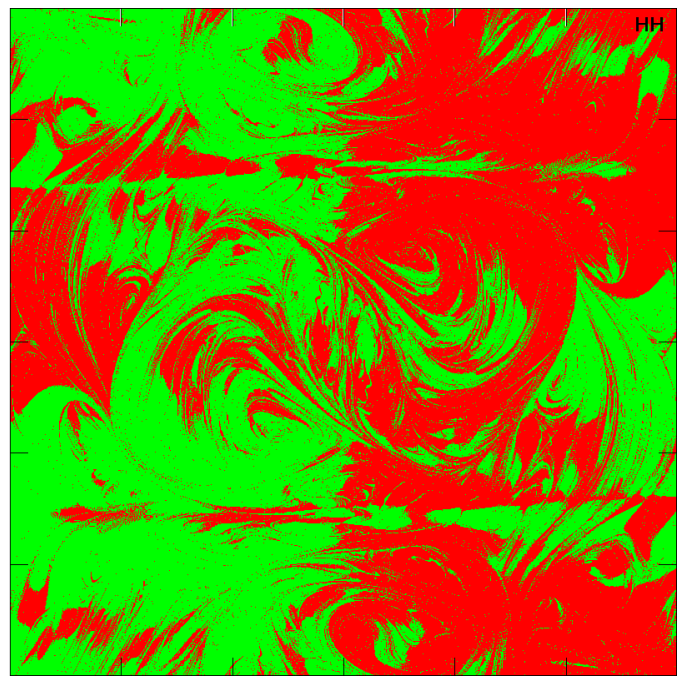

Figure 1: The sign of the local (time-dependent) value of the largest Lyapunov exponent for the Hoover-Holian oscillator is shown in the $(q, p, 0,0)$ plane. Because a mirror reflection of the dynamics ( perpendicular to the $q$ axis ) changes the signs of both $q$ and $p$ the Figure reveals an inversion symmetry. In the upper half plane red indicates a positive exponent and green a negative. The colors are reversed in the lower half plane. The probability density in the cross-sectional plane is a simple Gaussian in $q$ and $p$. Nevertheless the local Lyapunov exponents vary in a multifractal manner throughout the four-dimensional space, reflecting their sensitivity to bifurcations in their past histories. In Figures 1-4 both $q$ and $p$ range from -3 to +3 .

oscillations is time enough to indicate whether or not a chosen initial condition ( either from a grid or chosen randomly ) converges to a spectrum close to the unique long-timeaveraged Lyapunov spectrum. Estimates of these long-time-averaged exponents, after a time $t=40000000$ using a fourth-order Runge-Kutta integrator with an adaptive timestep, are as follows :

$$
\{\lambda\}_{\mathrm{HH}}=+0.068_{0},+0.000,-0.000,-0.068_{0} \text {; }
$$

in the Hoover-Holian case, and

$$
\{\lambda\}_{\mathrm{JB}}=+0.079_{7},+0.0000,-0.0000,-0.079_{7}
$$

in the Ju-Bulgac case, and

$$
\{\lambda\}_{\mathrm{MKT}}=+0.066_{5},+0.0000,-0.0000,-0.066_{5} \text {. }
$$




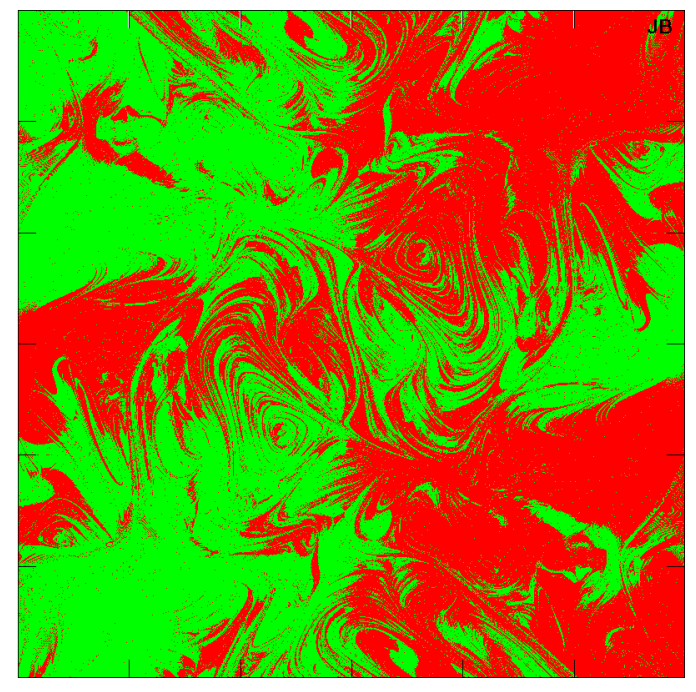

Figure 2: In the upper half plane red indicates a positive local Lyapunov exponent for the ergodic Ju-Bulgac oscillator model. The inversion symmetry ( with change of color ) is obeyed by all these time-reversible oscillator models. The probability density in the four-dimensional space is Gaussian in $q, p, \zeta$, and $\xi^{2}$ as it is also in the $(q, p, 0,0)$ plane displayed here.

in the Martyna-Klein-Tuckerman case.

The increase in the largest Lyapunov exponent with the number of quartic forces ( none for MKT, one for $\mathrm{HH}$, and two for JB ) suggests, as emphasized by Bulgac and Kusnezov ${ }^{\underline{9}}$, that these terms are particularly well-suited to promoting chaos and/or ergodicity. It seems likely that sextic forces, controlling $\left\langle p^{6}\right\rangle$, would have no particular advantages and would slow numerical work.

The probability densities for the three cases follow easily from the phase-space continuity equation. This makes it is easy to check for ergodicity by comparing relatively short-time estimates for the Lyapunov spectrum starting out with initial conditions chosen according to the stationary multivariable Gaussian distributions.

The ergodicity of the Martyna-Klein-Tuckerman oscillator was called into question by Patra and Bhattacharya in $2014^{14-16}$, based on apparent "holes" in a $(q, p, \zeta, \xi)=(q, p,-1,+1)$ double cross-section plane, the analog of a Poincaré plane for a three-dimensional flow problem. To resolve this question, the subject of the 2014 Ian Snook Prize ${ }^{15}$, we chose one million different initial conditions from the appropriate four-dimensional Gaussian distributions for 
the HH, JB, and MKT cases and determined that all of them were fully consistent with a unique chaotic spectrum. Each of these initial conditions was followed for one million fourth-order Runge-Kutta timesteps of 0.005 each.

The alternative possibility, a regular ( nonchaotic ) solution with all four of the long-timeaveraged Lyapunov exponents equal to zero, is easy to distinguish from the chaotic case. As an additional check the velocity moments generated by these three ergodic thermostat types likewise reproduce Gibbs' values for the second, fourth, and sixth velocity moments with five-figure accuracy :

$$
\left\langle p^{\{2,4,6\}}\right\rangle=\{1.00000,3.0000,15.000\} .
$$

By contrast, the motion equations for a Nosé-Hoover harmonic oscillator with a single friction coefficient $\zeta(t)$ and unit relaxation time ,

$$
\left\{\dot{q}=p ; \dot{p}=-q-\zeta p ; \dot{\zeta}=p^{2}-1\right\}
$$

are known not to be ergodic despite their simple three-dimensional Gaussian solution of the phase-space continuity equation. Choosing initial conditions from this stationary distribution $f_{\mathrm{NH}} \propto e^{-(1 / 2)\left(q^{2}+p^{2}+\zeta^{2}\right)}$ and measuring the tendency of a nearby initial condition to separate gives the largest Lyapunov exponent for the chosen initial condition, $\lambda_{1}=\langle(d / d t)(\ln (\delta r))\rangle$. Because this system is conservative and time-reversible the longtime-averaged Lyapunov spectrum is symmetric, $\left\{+\lambda_{1}, 0,-\lambda_{1}\right\}$, and sums to zero. 10000 initial conditions followed for a time $t=50000$ divide neatly into two groups :

$$
0.00002<\lambda_{1}<0.00014 ; 0.006<\lambda_{1}<0.017 \text {. }
$$

557 initial conditions correspond to a Lyapunov exponent significantly different to zero while 9443 correspond to nonchaotic toroidal solutions for which all three Lyapunov exponents vanish. Evidently roughly $6 \%$ of the stationary measure is chaotic ( with a positive timeaveraged largest Lyapunov exponent ). The remaining $94 \%$ consists of regular tori with a vanishing Lyapunov spectrum :

$$
\left\langle\lambda_{1}, \lambda_{2}, \lambda_{3}\right\rangle=\{0,0,0\}
$$

In the language of control theory the Nosé-Hoover equations control the time-averaged value of the kinetic temperature, $\left\langle\left(p^{2} / m k\right)\right\rangle \equiv T$. Subsequent work strongly suggests 


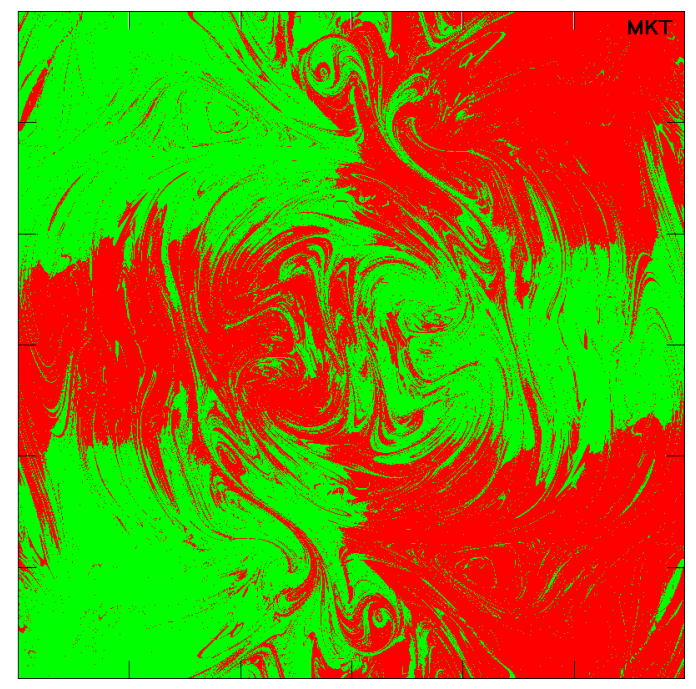

Figure 3: Red indicates a positive Lyapunov exponent in the upper half plane, negative in the lower. If the equations of motion are run backward ( by changing the righthandsides of all four equations ) the effect is to reflect the section shown here, changing the sign of $q$. Like the other models this Martyna-Klein-Tuckerman oscillator has a four-dimensional Gaussian distribution in its $(q, p, \zeta, \xi)$ phase space which the dynamics samples ergodically.

that an ergodic oscillator requires control of at least two moments, not just one. Such an approach requires four or more ordinary differential equations. Bulgac and Kusnezov $\underline{\underline{9}}$ as well as $\mathrm{Ju}$ and Bulgac ${ }^{10}$ added a fifth equation so as to be able to thermostat a free particle ( or a rotating and translating cluster of particles ) undergoing Brownian motion.

The four-dimensional phase-space continuity equation shows that both the MartynaKlein-Tuckerman and the Hoover-Holian thermostats are consistent with exactly the same four-dimensional Gaussian, $f_{\mathrm{HH}}=f_{\mathrm{MKT}}$. Unlike the Nosé-Hoover single-thermostat model we believe that the three two-thermostat models are all ergodic. We believe that this proposition has been thoroughly confirmed by the present work, confirming the chaos of one million independent initial conditions in each case. In view of the lack of other suitable entries in the 2014 competition, we have awarded ourselves the Snook Prize for this finding. It should be noted that all of these thermostat models, ergodic or not, are time-reversible, with the friction coefficients $(\zeta, \xi)$ and the momentum $p$ simply changing sign if the timeordered sequence of coordinate values $\{q(t)\}$ is reversed. For this reason the time-averaged 


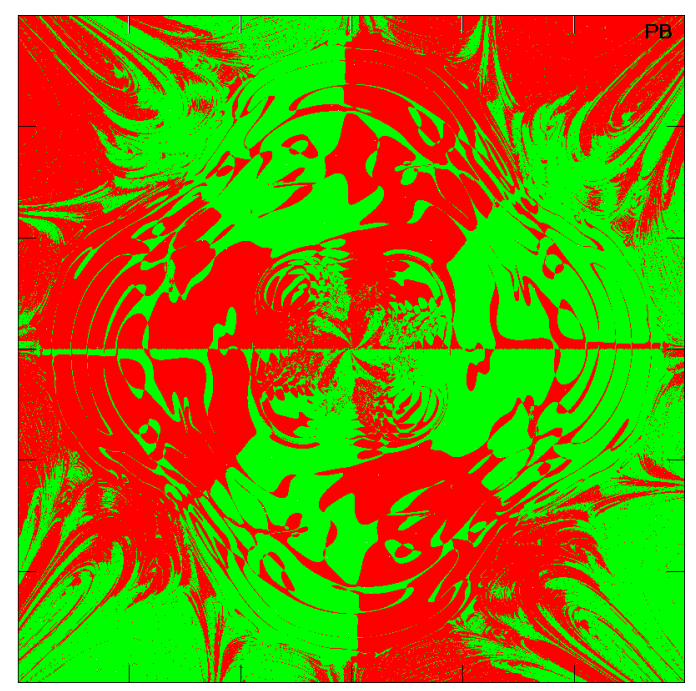

Figure 4: Red indicates a positive Lyapunov exponent in the upper half plane, negative in the lower. If the equations of motion are run backward by changing the righthandsides of all four equations the effect is to reflect the section shown here, changing the sign of $q$. Like the other models this Patra-Bhattacharya oscillator has a four-dimensional Gaussian distribution in its $(q, p, \zeta, \xi)$ phase space which the dynamics samples ergodically. Unlike the other models about half the PB measure is regular rather than chaotic.

Lyapunov spectrum is symmetric, with $\lambda_{1}+\lambda_{4}=\lambda_{2}+\lambda_{3}=\lambda_{2}=\lambda_{3}=0$. Despite the simple analytic nature of the phase-space distribution, the chaos implied by a positive $\lambda_{1}$ engenders an amazing complexity, singular in its spatial behavior, to which we turn next.

In exploring the $\mathrm{HH}, \mathrm{JB}$, and MKT ergodic oscillator solutions we uncovered an amazingly intricate multifractal structure most simply described in terms of the local largest Lyapunov exponent, $\lambda_{1}(t)$. The three phase-space distributions $\{f(q, p, \zeta, \xi)\}$ are all multidimensional Gaussians, of the form ,

$$
f(q, p, \zeta, \xi) \propto e^{-q^{2} / 2} e^{-p^{2} / 2} e^{-\zeta^{2} / 2} e^{-\xi^{n} / n}
$$

where $n$ is 2 for $\mathrm{HH}$ and MKT and 4 for JB. A closer look, using Lyapunov instability as a tool, reveals the interesting structures shown for the three thermostated oscillators in Figures 1-3 . For completeness, the corresponding Patra-Bhattacharya cross section is shown as Figure 4 .

For each of the three ergodic models we show the sign of the local Lyapunov exponent, 
$\lambda_{1}(t)$ at the location $(q, p, 0,0)$. The plots are analogous to a Poincare section for the more usual three-dimensional flows. At the equilibrium, $T=1$, these instability plots can be generated in either of two ways: [ i ] Whenever a $(q, p, 0,0)$ trajectory comes close to $(\zeta, \xi)=(0,0)$ plot the sign of the corresponding Lyapunov exponent at the location $(q, p)$; [ ii ] Starting at $(q, p, 0,0)$ integrate backwards in time for several hundred thousand timesteps, storing the backward trajectory. Then process the reversed trajectory data in the forward direction, with Lyapunov instability controlling a nearby constrained satellite trajectory, finding the local Lyapunov exponent at the chosen endpoint location $(q, p, 0,0)$. It turns out that these two methods are roughly comparable in cost. The second method has the advantage of providing more detail in regions where the measure is small. The precision shown in Figures 1-4 can be generated in simulations taking a day or two on a single processor. Method [ii ] above, reversing a stored trajectory, is particularly well-suited to parallel simulations. The Figures use color to indicate the sign of the local exponent.

\section{FRACTALS LURK IN THE SIMPLE GAUSSIAN DISTRIBUTIONS}

These cross sections have a fractal look. In fact they are. The reason for this was pointed out in our paper with Dennis Isbister $\underline{\underline{13}}$. As the "past" history of a point is being generated, backward from the "present", "bifurcations", where the direction of the trajectory is uncertain at the level of the numerical work limit the trustworthy scale of the Lyapunov exponent when the stored integration is reversed, going forward in time. For the megapixel details shown in Figures 1-4 a few hundred thousand fourth-order ( or fifth-order or adaptive ) Runge-Kutta timesteps suffice for reliable results at the resolution of the plotted sections. More detail can always be generated, at a cost exponential in the number of significant figures, by reducing the numerical error of the trajectory integration.

The cross sections shown in the Figures have inversion symmetry. As the source of this symmetry is not so obvious let us describe it. Consider a solution of the equations of motion going forward in time, $\left\{q(t), p(t), \zeta(t), \xi(t), \lambda_{1}(t)\right\}$. Viewed simultaneously in a mirror

perpendicular to the $q$ axis an observor sees $\left\{-q(t),-p(t), \zeta(t), \xi(t), \lambda_{1}(t)\right\}$. Evidently there is an inversion symmetry, with exactly the same Lyapunov exponent at two different values of $(q, p)$ :

$$
\lambda_{1}(+q,+p) \equiv \lambda_{1}(-q,-p) .
$$


This observation saves a factor of two in computer time. The two-dimensional cross sections where both of the friction coefficients vanish, $\zeta=\xi=0$ are the starting points for the backward integrations. The Figures indicate the magnitude of the largest Lyapunov exponent obtained at the original starting point from a reversed trajectory, with red positive and green negative as the local exponents varies with position. This is the case for positive $q$. The colors are reversed for negative coordinate values in order to eliminate the discontinuity that would otherwise occur at vanishing $q$.

As is usual the fluctuations in $\lambda_{1}(t)$ are an order of magnitude larger than its long-timeaveraged value. The variation of the local Lyapunov exponent is not quite continuous. If the local exponent is plotted along a line in the $(q, p)$ plane there are occasional jumps, present on all scales, in the exponent value. The fractal dimension accounts for the variation of the jump magnitudes as a function of resolution. In the MKT case ${ }^{13}$ the data along such a line gave a fractal dimension of 1.69 rather than 1.00. Studies of the jumps along such lines reveal their fractal structure, seen here for the first time in two dimensions.

\section{THERMOSTATING CONFIGURATIONAL TEMPERATURE ?}

All three of the two-thermostat HH, JB, and MKT models for heat-bath control of a harmonic oscillator are ergodic, Because of this it was a complete surprise that Patra and Bhattacharya's control of both the kinetic and the configurationalle oscillator temperatures, using two thermostat variables, was unsuccessful, leading to a mix of regular and chaotic solutions. Their model was :

$$
\left\{\dot{q}=p-\xi q ; \dot{p}=-q-\zeta p ; \dot{\zeta}=p^{2}-1 ; \dot{\xi}=q^{2}-1\right\} \rightarrow\left\langle p^{2}, q^{2}\right\rangle=(1,1)[\mathrm{PB}] .
$$

The stationary phase-space distribution function for the PB equations is the same as that characterizing the $\mathrm{HH}$ and MKT models :

$$
f_{\mathrm{HH}}=f_{\mathrm{MKT}}=f_{\mathrm{PB}}=(2 \pi)^{-2} e^{-q^{2} / 2} e^{-p^{2} / 2} e^{-\zeta^{2} / 2} e^{-\xi^{2} / 2} .
$$

But unlike the HH and MKT equations the Patra-Bhattacharya model fails the Lyapunovexponent test for ergodicity. About half the initial conditions chosen from the fourdimensional Gaussian distribution give regular rather than chaotic solutions.

Although controlling coordinate moments like $\left\langle q^{2}, q^{4}, q^{6} \ldots\right\rangle$ may seem unphysical Landau and Lifshitz showed ( in the 1951 Russian edition of their Statistical Physics ) that 
what later came to be termed a "configurational temperature" $\underline{20}$, based on a mean-squared force, can be derived from Gibbs' canonical distribution. A single integration by parts, in the canonical ensemble, relates the mean-squared force to the curvature of the potential through the temperature $T$ :

$$
k T \int\left[\nabla^{2} \mathcal{H}\right] e^{-\mathcal{H} / k T} d q \equiv \int\left[(\nabla \mathcal{H})^{2}\right] e^{-\mathcal{H} / k T} d q
$$

So control of $\left\langle q^{2}\right\rangle$ can be viewed as regulating the fluctuations in the forces. This concept is more appealing than coordinate control. But the lack of a physical "thermometer" capable of measuring force-based temperature is a disabling disadvantage of configurational temperature. The possibility of divergent or negative values of the instantaneous configurational temperature are further caution flags. The Campisi "thermostat" is an example of both defects :

$$
\mathcal{H}=\left(p^{2} / 2\right)+(T / 2) \ln \left(q^{2}\right) \rightarrow\{\dot{q}=p ; \dot{p}=-(T / q)\}
$$

Formally, Campisi dynamics is consistent with a Gaussian momentum distribution. Although at first glance appealing, this simple model exhibits a negative configurational temperature, $-T$, in one dimension and a divergent one in two 21 . Disagreement between the kinetic and configurational temperatures is a clear violation of the Zeroth Law of Thermodynamics, to which we turn next.

\section{COUPLED THERMOSTATS-THE ZEROTH LAW OF THERMODYNAMICS}

To what extent do these minimalistic thermostats, described by one or two additional control variables, exhibit the characteristic properties of macroscopic thermal baths? An essential characteristic of such baths is their consistency with the Zeroth Law of Thermodynamics. Two "good" thermal baths, both at a temperature $T$, should, when coupled together or to another canonical equilibrium system at the same temperature $T$, remain at that common temperature without modifying Gibbs' equilibrium distribution in either bath or in another equilibrium system.

For our thermostated oscillators this desirable property can be tested by coupling pairs of heat baths together with a simple Hooke's-Law spring. The modification of the equations of motion for oscillator number 1 and oscillator number 2, both at unit temperature, are as 
follows :

$$
\phi_{12}=\left(q_{1}-q_{2}\right)^{2} / 2 \rightarrow \dot{p}_{1}=\dot{p}_{1}(T=1)+q_{2}-q_{1} ; \dot{p}_{2}=\dot{p}_{2}(T=1)+q_{1}-q_{2} .
$$

It is easy to verify that the two Nosé-Hoover oscillators, coupled together by a spring, have nonergodic solutions, corresponding to a six-dimensional analog of the tori making up most of the single-oscillator phase space. One such solution occurs with initial values of the two oscillator momenta $\left(p_{1}, p_{2}\right)=(0.99,1.01)$. Accordingly we restrict our detailed investigation to the three ergodic two-thermostat heat baths to see whether or not they can obey the Zeroth Law.

All the six combinations of like and unlike pairs of ergodic two-thermostat oscillators :

$$
[\mathrm{HH}+\mathrm{HH}, \mathrm{HH}+\mathrm{JB}, \mathrm{HH}+\mathrm{MKT}, \mathrm{JB}+\mathrm{JB}, \mathrm{JB}+\mathrm{MKT}, \mathrm{MKT}+\mathrm{MKT}],
$$

provide chaotic eight-dimensional regions in phase space with symmetric Lyapunov spectrum. This symmetry implies ergodicity. Figure $\mathbf{5}$ shows both the spectra and the spectral sums ( which give the growth rates of phase-space hyperspheres as functions of their dimensionality ). Even though there are relatively small but significant differences between the $\mathrm{HH}, \mathrm{JB}$, and MKT Lyapunov exponents pairing any two of these systems together ( with a Hooke's-Law spring ) gives no indication of the dissipation which would result if one phasespace bath were to grow at the expense of the other. We conclude from these six examples that the HH, JB, and MKT heat baths all obey the Zeroth Law of Thermodynamics, quite a good outcome for dynamical systems which represent a thermodynamic heat bath with the low cost of only four phase-space dimensions.

The largest Lyapunov exponent in these eight-dimensional problems varies from 0.20 for two coupled MKT oscillators to 0.23 for two coupled JB oscillators. The exponent for two HH oscillators lies in between. Evidently the quartic forces in the Ju-Bulgac and Hoover-Holian thermostats are slightly more effective in promoting chaos than are the cubic MartynaKlein-Tuckerman forces. This same conclusion follows from the time required to generate the structures seen in Figures 1-4. The additional chaos comes at the ( rather small ) price of an increased stiffness in the differential equations. If any of the three ergodic thermostats were not ergodic we would expect to see a dissipative strange attractor result in coupling it to an ergodic heat bath. In all six pairings of the equilibrium ergodic thermostats there is no evidence of dissipation or reduced phase-space dimensionality. Evidently all three $(\zeta, \xi)$ 


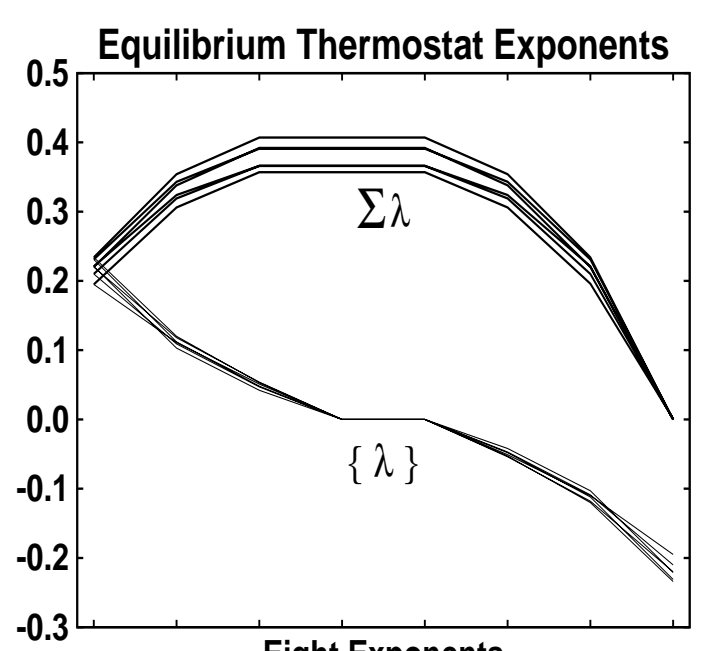

Eight Exponents

Figure 5: The eight equilibrium Lyapunov exponents describing pairs of ergodic thermostats linked by a Hooke's-Law spring exhibit pairing when time averaged. The JB thermostats exhibit slightly larger Lyapunov exponents due to the quartic terms in their equations of motion. The summed spectra shown in the six upper lines give the growth rates of 1-, 2-, 3-, .. 8-dimensional phase volumes. The last sum is precisely zero as the equilibrium equations of motion conserve phase volume when time-averaged.

thermostats are good heat baths from the standpoint of the Zeroth Law of Thermodynamics. Coupling pairs of thermostated oscillators with a harmonic coupling reveals that all three of the ergodic models $\{\mathrm{HH}, \mathrm{JB}, \mathrm{MKT}\}$ remain ergodic when coupled and also provide the complete canonical distribution.

\section{NONEQUILIBRIUM SIMULATIONS}

The similar equilibrium behavior of the HH, JB, and MKT thermostats suggests a further comparison for simple heat-flow problems in which two coupled thermostats have different temperatures, leading to hot-to-cold heat flow and dissipation. Dissipation is reflected in phase space by the formation of strange attractors with a fractional dimensionality reduced below the equilibrium value. In the linear-response regime the six two-bath possibilities must necessarily agree with Green and Kubo's theory as all the two-constant heat baths reproduce Gibbs' canonical $(q, p)$ distribution at equilibrium. Let us be adventurous and treat instead the relatively far-from-equilibrium coupling of two thermostats with thermostat 1 at half 


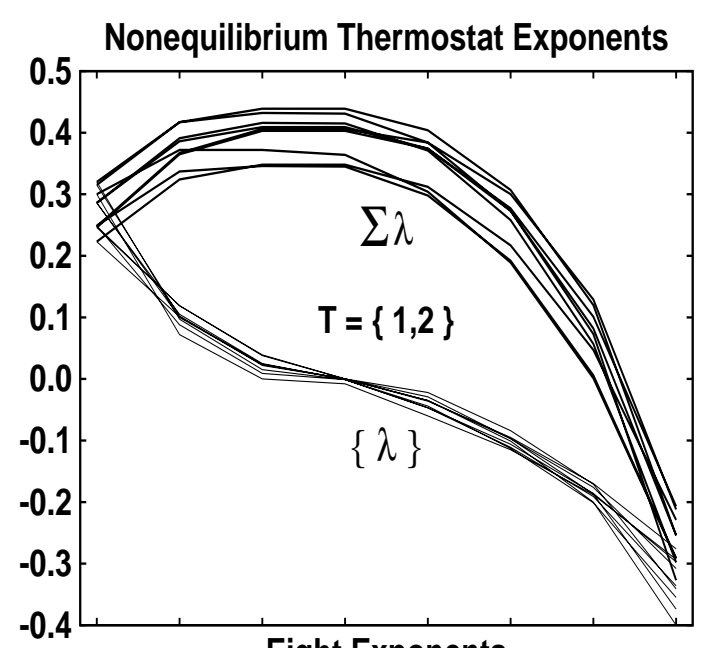

Eight Exponents

Figure 6: The eight exponents describing the coupling of ergodic thermostats with markedly different temperatures, 1 and 2. Spectra for all nine combinations of thermostats are shown. The hot-to-cold heat current dissipates phase volume. On the average the phase volume approaches zero ( a strange attractor ) as $\exp \left[\sum \lambda t\right] \simeq e^{-0.25 t}$ despite the time-reversible nature of all the equations of motion.

the temperature of thermostat 2 :

$$
\dot{p}_{1}=\dot{p}_{1}(T=1)+q_{2}-q_{1} ; \dot{p}_{2}=\dot{p}_{2}(T=2)+q_{1}-q_{2}
$$

Just as before all the masses, force constants, and relaxation times are chosen equal to unity.

The far-from-equilibrium Lyapunov data corresponding to the nine combinations of thermostats, with temperatures of 1 and 2, are shown in Figure $\mathbf{6}$, both individually and as sums. The broad maximum corresponding to sums of three or four exponents indicates that the maximum growth rate in phase space applies to four-dimensional volumes. The summed-up spectrum crosses zero near an abscissa value of 7 indicating that the dimensionality loss for this far-from-equilibrium problem is on the order of 1 . Considerably larger losses, comparable to the number of particles, occur in " $\phi^{4}$-model" chain simulations where each of a few dozen particles is tethered to its lattice site with a cubic restoring force ${ }^{7}$.

Closer to equilibrium, with cold and hot temperature of 1.0 and 1.1, all nine thermostat combinations have a Lyapunov sum of order -0.01 while temperatures of 1.0 and 2.0 provide dissipative sums in the range from -0.20 to -0.30 . Compare Figures 6 and 7 . Just as in heat conduction according to Fourier's Law, we would expect a linear-response dissipation 


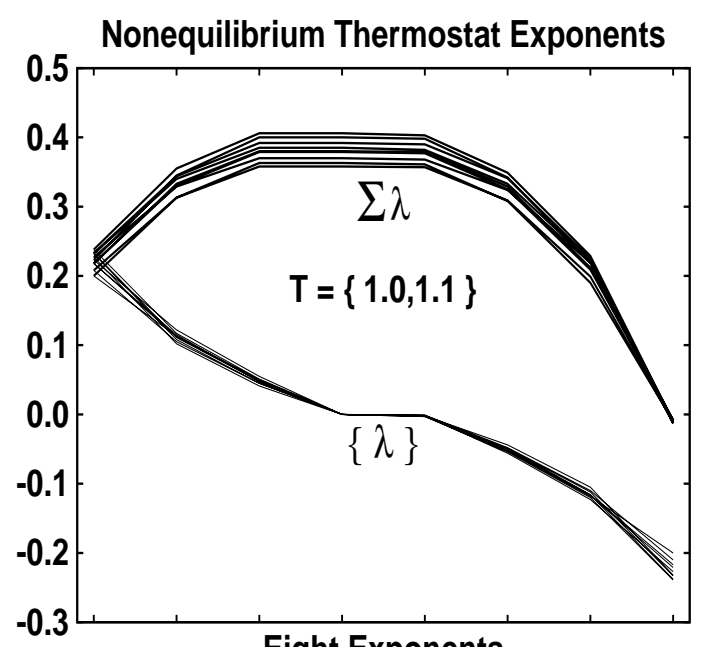

Eight Exponents

Figure 7: The eight exponents describing the coupling of ergodic thermostats with similar temperatures, 1 and 1.1. All nine combinations are shown. The hot-to-cold heat current could be estimated with linear response theory and is essentially the same for all three thermostat types because the equilibrium phase-space distribution is the same Gaussian for all three thermostat types, $f(q, p) \propto e^{-q^{2} / 2} e^{-p^{2} / 2}$.

quadratic in the temperature difference driving the flow, varying as $\left(T_{\text {hot }}-T_{\text {cold }}\right)^{2}$.

Loss of phase-space volume corresponds to thermodynamic dissipation (through Gibbs' relationship of phase volume to entropy, $S=k \ln \Omega$, where $\Omega$ is phase volume or number of states ). Coupling two baths, with the cooler bath first, gives the following "entropy production rates" :

$$
\begin{gathered}
\mathrm{HH}+\mathrm{HH}: 0.322 ; \mathrm{HH}+\mathrm{JB}: 0.254 ; \mathrm{HH}+\mathrm{MKT}: 0.213 ; \\
\mathrm{JB}+\mathrm{HH}: 0.299 ; \mathrm{JB}+\mathrm{JB}: 0.253 ; \mathrm{JB}+\mathrm{MKT}: 0.207 ; \\
\mathrm{MKT}+\mathrm{HH}: 0.291 ; \mathrm{MKT}+\mathrm{JB}: 0.290 ; \mathrm{MKT}+\mathrm{MKT}: 0.229 .
\end{gathered}
$$

We used quote marks above to remind the reader that simply summing the spectrum is not the same as averaging the dissipation for the cold and hot thermostats.

Dissipation can also be reckoned in terms of the phase-space dimensionality of the dissipative strange attractor, using the Kaplan-Yorke linear interpolation between the last positive dimension and the first negative one. For the nine heat-bath pairings the dimensionalities are :

$$
\mathrm{HH}+\mathrm{HH}: 7.19 ; \mathrm{HH}+\mathrm{JB}: 7.32 ; \mathrm{HH}+\mathrm{MKT}: 7.38 ;
$$




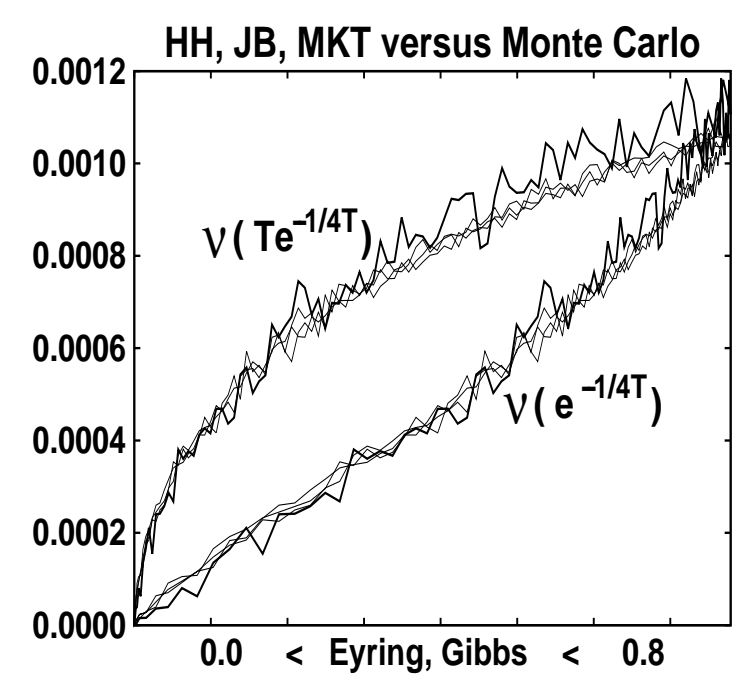

Figure 8: Coupling a Mexican Hat potential with twin well depths of 0.25 at $q= \pm 1$ to any of the three ergodic heat baths provides a mechanism for jumping over the barrier. The simulations shown here indicate that all three baths provide essentially the same temperature-dependent jump frequency $\nu$. The dependence is nearly linear when the coupling is small $(\kappa=1 / 10)$ and the abscissa is Gibbs': $e^{-1 / 4 T}$. Eyring's description ( the upper three curves ) is shown where the abscissa is now $T e^{-1 / 4 T}$. Gibbs' better approximates an Arrhenius straight line. Monte Carlo simulation of Mexican Hat dynamics, with a maximum jump length of $0.009 \sqrt{T}$, results in the heavier line plotted in addition to the results for the three heat-bath models.

$$
\begin{gathered}
\mathrm{JB}+\mathrm{HH}: 7.16 ; \mathrm{JB}+\mathrm{JB}: 7.25 ; \mathrm{JB}+\mathrm{MKT}: 7.33 ; \\
\mathrm{MKT}+\mathrm{HH}: 7.02 ; \mathrm{MKT}+\mathrm{JB}: 7.01 ; \mathrm{MKT}+\mathrm{MKT}: 7.17 .
\end{gathered}
$$

These results should provide good benchmarks for nonlinear theories of transport.

\section{JUMPS - EQUILIBRIUM MEXICAN HAT POTENTIAL SIMULATIONS}

The double-well "Mexican Hat" potential ${ }^{8}$,

$$
\phi(q)=-\left[\frac{q^{2}}{2}\right]+\left[\frac{q^{4}}{4}\right] \longrightarrow \phi( \pm 1)=-\left[\frac{1}{4}\right] ; \phi(0)=0,
$$

has a barrier between its two minima of height (1/4). At a temperature of unity, where the barrier is negligible, simulations of the Hat potential coupled to the HH, MKT, and JB thermostats result in excellent agreement with Gibbs' canonical distribution. At sufficiently low temperatures we expect that the frequency of successful jumps over the barrier will 
decline, as would also be the case using the Metropolis, Rosenbluths, and Tellers' Monte Carlo scheme with a reasonable jump length, perhaps of order 0.01 .

A straightforward "weak coupling" of the two-minimum Hat potential to any one of the ergodic oscillator heat baths ,

$$
\Phi\left(q_{\text {Bath }}, q_{\text {Hat }}\right)=\frac{1}{20}\left(q_{\text {Bath }}-q_{\text {Hat }}\right)^{2},
$$

leads to "jump" frequencies that vary roughly as Gibbs' canonical barrier weight, $e^{-1 / 4 T}$. Eyring's model, as mentioned in the Wikipedia article on "Henry Eyring ( chemist)", and with a jump frequency varying as $T e^{-1 / 4 T}$, is a relatively poor description and corresponds

to the upper curves in the Figure 8 . All three ergodic thermostat models behave similarly, as is shown in triplets of narrower lines in Figure 8 .

Another possibility for modelling jumps is to use the Metropolis, Rosenbluths, and Tellers' Monte Carlo method. By adusting the Monte Carlo trial steplength the two approaches, dynamical and Monte Carlo, can be made to correspond. We have compared the number of jumps over the barrier in billion-jump simulations with maximum jump length $d q=0.009 \sqrt{T}$ to dynamical simulations with timestep 0.005, where in all these latter dynamical cases the Hat potential is coupled to an $\mathrm{HH}$, MKT, or JB thermostat with temperatures varying from 0.010 to 1.00 , as shown in Figure 8 .

\section{SUMMARY}

The Lyapunov spectra for three varieties of time-reversible deterministic oscillator thermostats show that all three of them reproduce an extended ( four-variable ) form of Gibbs' canonical ( two-variable ) distribution ,

$$
f(q, p)=e^{-q^{2} / 2} e^{-p^{2} / 2} /(2 \pi) .
$$

The HH and MKT equations have Gaussian distributions for $\zeta$ and $\xi$ while the JB distribution is Gaussian in $\zeta^{2}$ and $\xi$. All three approaches are sufficiently robust to thermostat a harmonic oscillator. The three are also consistent with the Zeroth Law of Thermodynamics in the sense that any pair of them at a common temperature $T$ generates Gibbs' canonical distribution for that temperature for both thermostats.

This set of ergodic thermostats represents a good toolkit for simulations with a few degrees of freedom as it gives an idea of the dependence of dissipation on the chosen form of 
the thermostat. With many degrees of freedom, where ergodicity is not an issue [ Poincaré recurrence takes forever so that the size of fluctuations determines the usefulness of the results ] any of these thermostats, as well as the single thermostat Nosé-Hoover model, are likely equally useful.

We have considered two nonergodic time-reversible thermostats, the Patra-Bhattacharya and Nosé-Hoover, which reproduce the specified kinetic temperature but do not reproduce all of Gibbs' canonical distribution. When the PB or NH thermostats are coupled to the HH, JB, or MKT thermostats two qualitatively different results occur: [ 1 ] a dissipative strange attractor in the case of the PB model and [2 ] a conservative integer-dimensional chaotic sea when coupled to the Hamiltonian-based Nosé-Hoover oscillator. These results are similar for each of the three ergodic thermostats. In the $\mathrm{PB}$ case the dissipation is an indicator of the nonHamiltonian nature of the dynamics. Any of the ergodic thermostats, HooverHolian, Ju-Bulgac, Martyna-Klein-Tuckerman, can be used successfully in both equilibrium and nonequilibrium simulations.

The first two of these can also be applied in nonequilibrium simulations where it is desired to specify the kinetic temperature of selected degrees of freedom. Though there is no problem in solving the MKT equations for nonequilibrium problems Brad Holian pointed out long ago $\frac{18}{}$ that this thermostat fails to return its target temperature due to the nonzero correlation linking the two MKT friction coefficients, $\langle\zeta \xi\rangle_{\mathrm{MKT}}^{\text {noneq }} \neq 0$.

This relatively thorough investigation of ergodicity, based on one million independent initial conditions, should settle ( at least from the numerical, as opposed to analytical, viewpoint ) the question of the ergodicity of the Martyna-Klein-Tuckerman oscillator. The apparent "holes" in that oscillator's cross section are still a small "puzzle". A second such "puzzle" is the nature of the local Lyapunov exponents. Their intricate multifractal Lyapunov structure is well concealed within an innocent Gaussian distribution.

From the standpoint of dynamical-systems theory ( as opposed to thermodynamics ) we wish to emphasize the amazing nature of the chaos hidden within the simple ergodic Gaussian distributions. The smoothness of the distributions and the good convergence of their moments conceals the fractal nature of their chaos, as shown in Figure 1 through 3 . We recommend all three systems for further studies. The $(\zeta=\xi=0)$ plane is only a single choice among the many possible. Despite their fractal nature the cross-sectional values are well-behaved, accessible, and reproducible from the numerical standpoint. It is encouraging 
for the future that work originally chosen to settle some ergodicity questions turned out to generate a swarm of other problems still needing more detailed understanding.

\section{ACKNOWLEDGMENTS}

We thank Carol Hoover, Aurel Bulgac, and Baidurya Bhattacharya, for their able help.

1 W. G. Hoover and B. J. Alder, "Studies in Molecular Dynamics. IV. The Pressure, Collision Rate, and Their Number Dependence for Hard Disks", Journal of Chemical Physics 46, 685-691 (1967).

2 S. Nosé, "A Unified Formulation of the Constant Temperature Molecular Dynamics Methods", The Journal of Chemical Physics 81, 511-519 (1984).

3 S. Nosé, "A Molecular Dynamics Method for Simulations in the Canonical Ensemble", Molecular Physics 52, 191-198 (1984).

4 W. G. Hoover, "Canonical Dynamics: Equilibrium Phase-Space Distributions", Physical Review A 31, 1695-1697 (1985).

5 H. A. Posch, W. G. Hoover, and F. J. Vesely, "Canonical Dynamics of the Nosé Oscillator: Stability, Order, and Chaos" Physical Review A 33, 4253-4265 (1986).

6 Wm. G. Hoover and C. G. Hoover, Time Reversibility, Computer Simulation, Algorithms, Chaos ( World Scientific, Singapore, 2012 ).

7 Wm. G. Hoover and C. G. Hoover, Simulation and Control of Chaotic Nonequilibrium Systems ( World Scientific, Singapore, 2015 ).

8 D. Kusnezov, A. Bulgac and W. Bauer, Annals of Physics (NY) 204, 155-185 (1990).

9 A. Bulgac and D. Kusnezov, "Canonical Ensemble Averages from Pseudomicrocanonical Dynamics", Physical Review A 42, 5045-5048 (1990).

10 N. Ju and A. Bulgac, "Finite-Temperature Properties of Sodium Clusters", Physical Review B 48, 2721-2732 (1993).

11 I. Shimada and T. Nagashima, "A Numerical Approach to Ergodic Problems of Dissipative Dynamical Systems, Progress of Theoretical Physics 61, 1605-1616 (1979) .

12 G. Benettin, L. Galgani, A. Giorgilli, and J. M. Strelcyn, Lyapunov Characteristic Exponents 
for Smooth Dynamical Systems and for Hamiltonian Systems; a Method for Computing All of Them. Part 1: Theory, Meccanica 15, 9-20 (1980) .

13 Wm. G. Hoover, C. G. Hoover, and D. J. Isbister, "Chaos, Ergodic Convergence, and Fractal Instability for a Thermostated Canonical Harmonic Oscillator", Physical Review E 63, 026209 (2001).

14 P. K. Patra and B. Bhattacharya, "Non-Ergodicity of Nosé-Hoover Chain Thermostat in Computationally Achievable Time", Physical Review E 90, 043304 (2014) = ar $\chi$ iv : 1407.2353 (2014).

15 Wm. G. Hoover and C. G. Hoover, "Ergodicity of a Time-Reversibly Thermostated Harmonic Oscillator and the 2014 Ian Snook Prize", Computational Methods in Science and Technology 20, 87-92 (2014).

16 P. K. Patra and B. Bhattacharya, "Improving the Ergodic Characteristics of Thermostats Using

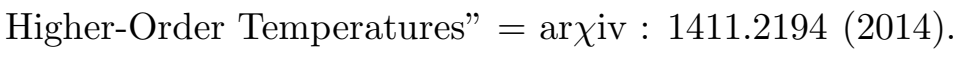

17 G. J. Martyna, M. L. Klein, and M. Tuckerman, "Nosé-Hoover Chains: the Canonical Ensemble via Continuous Dynamics", The Journal of Chemical Physics 97, 2635-2643 (1992).

18 W. G. Hoover and B. L. Holian, "Kinetic Moments Method for the Canonical Ensemble Distribution", Physics Letters A 211, 253-257 (1996).

19 P. K. Patra and B. Bhattacharya, "A Deterministic Thermostat for Controlling Temperature Using All Degrees of Freedom", Journal of Chemical Physics 140064106 (2014).

20 C. Braga and K. P. Travis "A Configurational Temperature Nosé-Hoover Thermostat", Journal of Chemical Physics 123, 134101 (2005).

21 D. Sponseller and E. Blaisten-Barojas, "Failure of Logarithmic Oscillators to Thermostat Small Atomic Clusters", Physical Review E 89, 021301(R) (2014). 